\title{
Lower gastrointestinal bleeding as a result of fistula between the iliac artery and sigmoid colon in patient with advanced testicular cancer
}

Vanessa Prado dos Santos, Álvaro Razuk Filho, Valter Castelli Júnior, Roberto Augusto Caffaro

Instituto de Humanidades Artes e Ciências Professor Milton Santos, Bahia Federal University (VPS), Bahia; Department of Vascular Surgery, Medical Sciences Faculty of Santa Casa de Sao Paulo (ARfilho, VCJr, RAC), Sao Paulo, Brazil

\section{ABSTRACT}

Fistula between arteries and the gastrointestinal tract are a rare cause of gastrointestinal bleeding, but potentially fatal. The recognition and early treatment can modify the patient prognosis. We report a case of a patient with previous surgery for seminoma of cryptorchidic testicle, with massive lower gastrointestinal bleeding. We performed the diagnosis and surgical treatment of the fistula between left external iliac artery and sigmoid colon. The patient was successfully treated by external iliac artery ligation and left colectomy.

\section{ARTICLE INFO}

Key words:

Gastrointestinal Hemorrhage; Seminoma; Fistula; General Surgery

Int Braz J Urol. 2013; 39: 747-51

Submitted for publication: June 27, 2012

Accepted after revision: May 25, 2013

\section{INTRODUCTION}

Main causes of massive lower gastrointestinal bleeding include colon diverticular disease and vascular malformations (1). Right colon is most frequently the involved organ in bleeding and diagnosis is often performed using endoscopic and image exams (1). Fistulae between arteries and gastrointestinal tract are a rare cause of gastrointestinal bleeding, and most frequently in literature include aorto-enteric fistulae $(2,3)$ secondary to aorta surgeries, with communication between proximal anastomosis and duodenum $(2,3)$. Diagnosis of aorto-enteric fistulae, primary (very rare) and secondary, is complex. Morbidity and mortality are high when associated with bleeding and sepsis (2-4).

The objective of the present paper is to report a case of fistula between extern iliac artery and sigmoid colon leading to massive intestinal bleeding in a patient with seminoma.

\section{CASE REPORT}

A male patient 39 years old attended the Emergence Room of Santa Casa de Misericórdia in Sao Paulo, Brazil, with enterorrhagia lasting 8 hours. He reported a slight bleeding during evacuation in 
the last 7 days. He had been submitted to left orchiectomy 3 months ago due to neoplasia of criptorchidic testicle. Pathological exam showed seminoma of left testicle and the patient had also been submitted to three sessions of chemotherapy after orchiectomy. During physical exam, the patient was pale, tachycardic, eupneic, with a blood pressure of $80 \times 40 \mathrm{mmHg}$. Hematocrit was 21.3\% (normal $36-47 \%)$. He was hemodynamically stabilized and submitted to arteriography due to lower intestinal bleeding, in order to identify the source and localization of the bleeding. Due to massive bleeding, scintigraphy was not performed. Initial aortography showed extravasation of contrast media in the left external iliac artery, compatible with pseudoaneurism, and probable communication (fistula) with large intestine (Figure-1).

Figure 1 - Arteriography of the patient, showing leakage of contrast medium at the left external iliac artery, compatible with pseudoaneurism (arrow to the left), with probable fistula to the colon (arrow down).

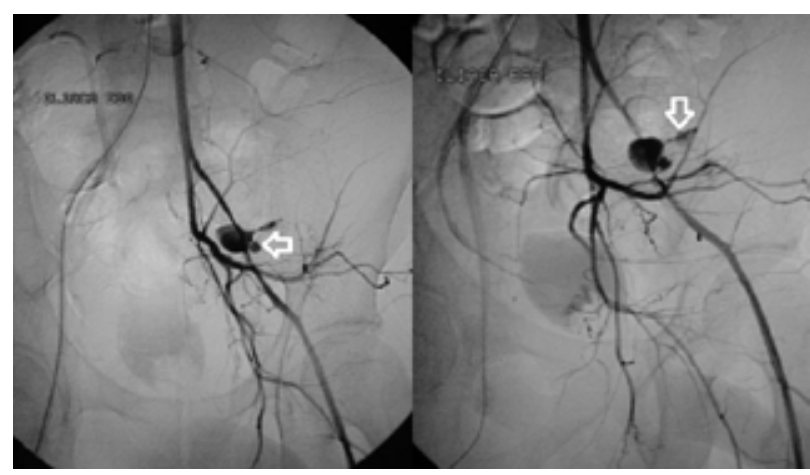

Following arteriography, patient was immediately submitted to surgical treatment by vascular and general surgeons. The patient was placed in right lateral decubitus and a left extraperitoneal access was obtained in order to rapidly control the bleeding from left external iliac artery, the source of the bleeding. However, an intense periarterial block made the access to left external iliac artery very difficult, in order to control bleeding. So, a right inguinotomy was performed, in order to dissect common femoral artery and to introduce a 7 Fr Fogarty ${ }^{\odot}$ until aorta, where the balloon was inflated. This maneuver allowed the control of the arterial bleeding. In order to identify the left external iliac artery in the middle of the intense local block, a left inguinotomy was also performed and it was also introduced a 7F Fogarty ${ }^{\odot}$ catheter through left common femoral artery. This catheter allowed the identification of all left external iliac artery that showed several lesion throughout the pelvic block and also allowed total control of bleeding (Figure-2).

In between the inflammatory block of left iliac fossa it was possible to visualize sigmoid colon mucosa right to the region of the arterial rupture (Figure-2). Due to gross contamination of the abdominal cavity, it was performed ligature of left external iliac artery (proximal and distal stumps) and sigmoidectomy followed by colostomy. Internal left iliac artery was pervious and with good caliber and kept a good perfusion of left leg during arterial clamping, allowing arterial ligature. Patient evolved without complications and was discharged after 10 days. There were no signs of left leg ischemia and

Figure 2 - Intra-operatory image showing extensive destruction or the arterial wall of the left external iliac artery, containing the 7FR Fogarty@ catheter (arrow down) and visualization of the sigmoid mucosa (arrow up) (forceps).

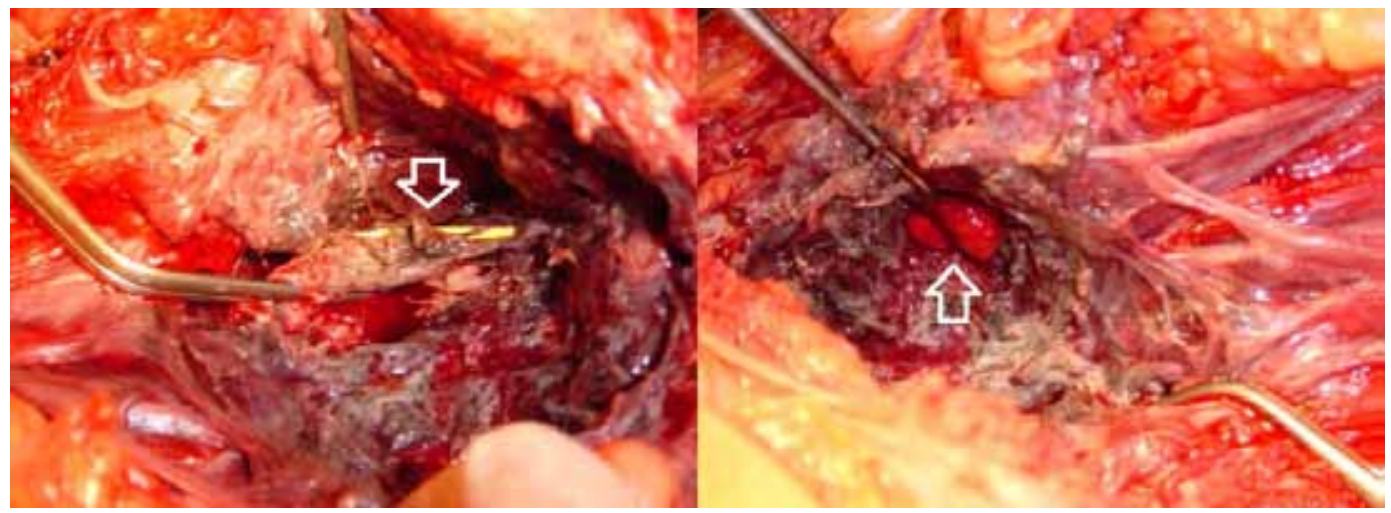


the patient was redirected to oncologic treatment. Pathological exam of the surgical specimen showed inflammatory process with fistula to large intestinal and lymphonodal reaction lymphoid hyperplasia.

\section{DISCUSSION}

There are very few reports in literature concerning gastrointestinal bleeding related to seminoma. Leslie et al. related a case of a patient with seminoma with thrombosis of inferior vena cava and melena, due to tumor infiltration of duodenum (5). Lymphatic dissemination of seminoma was reported by Miller et al. (6), causing retroperitoneal mass and duodenal ulcer and gastrointestinal bleeding. In that case report, the clinical presentation was different, since there was a fistula between artery and sigmoid colon, with massive intestinal bleeding and extensive arterial wall destruction. The patient was kept under oncologic treatment (that was initiated two months ago) despite absence of tumor in pathological exams. Rupture of external iliac artery was reported in two patients with advanced ovarian tumor, after 11 and 12 days of post-operatory of pelvic lymphadenectomy (7). Both patients were submitted to ligature of external iliac artery without ischemic complications of the left leg (7). Ligature of external iliac artery was also reported in a patient with anastomotic pseudoaneurism after renal transplantation (8). This patient was also submitted to ligature of external iliac artery without left leg ischemia and complications. Possibly a wide collateral arterial network among the arteries of thorax, pelvis and lower limbs allows refill of femoral arteries in case of occlusion of the aorto-iliac sector $(9,10)$. The internal iliac artery of this patient had a wide caliber and had not atherosclerotic lesions, and the ligature of the external iliac artery was well tolerated.
Classically, the surgery of fistulae between arteries and the gastrointestinal tract comprises one arterial ligature and extra-anatomic revascularizations due to local contamination (2-4). More recently, endovascular treatment of aorto-enteric fistulae has been proposed in literature, but when the arterial communication is with the colon, the risk of infection and sepsis usually indicates the need of conventional surgery (3). The time between the first bleeding of an aorto-enteric fistulae, called sentinel, and the massive bleeding usually takes hours or even months, but the correct diagnosis during the first bleeding improves prognosis (4). Although arteriography presents less sensitivity than scintigraphy to localize the source of intestinal bleeding, a positive exam is able to predict which patients will need surgical treatment (1). During massive intestinal bleeding, the identification of the localization of the bleeding using arteriography allows segmental intestinal resection, and better outcome (1). In the present report, the massive intestinal bleeding with hemodynamic alteration lead us to perform an initial and precocious angiography, that correctly identified the source and place of bleeding, allowing an efficient surgical treatment.

\section{CONFLICT OF INTEREST}

None declared.

\section{REFERENCES}

1. Leitman IM, Paull DE, Shires GT 3rd: Evaluation and management of massive lower gastrointestinal hemorrhage. Leitman IM, Paull DE, Shires GT 3rd. Ann Surg. 1989; 209: 175-80.

2. Baril DT, Carroccio A, Ellozy SH, Palchik E, Sachdev U, Jacobs TS, et al.: Evolving strategies for the treatment of aortoenteric fistulas. J Vasc Surg. 2006; 44: 250-7. 
3. Burks JA Jr, Faries PL, Gravereaux EC, Hollier LH, Marin ML: Endovascular repair of bleeding aortoenteric fistulas: a 5-year experience. J Vasc Surg. 2001; 34: 1055-9.

4. Song $Y$, Liu $Q$, Shen $H$, Jia X, Zhang $H$, Qiao L: Diagnosis and management of primary aortoenteric fistulas--experience learned from eighteen patients. Surgery. 2008; 143: 43-50.

5. Leslie JA, Stegemann L, Miller AR, Thompson IM: Metastatic seminoma presenting with pulmonary embolus, inferior vena caval thrombosis, and gastrointestinal bleeding. Urology. 2003; 62: 144.

6. Miller TT, Mendelson DS, Wu LT, Halton KP: Seminoma of the testis presenting as an ulcerating mass of the duodenum. Clin Imaging. 1992; 16: 201-3.

7. Fotopoulou C, Neumann U, Kraetschell R, Lichtenegger W, Sehouli J: External iliac artery ligation due to late postoperative rupture after radical lymphadenectomy for advanced ovarian cancer--two case reports. Eur J Gynaecol Oncol. 2010; 31: 198-200.

8. Silva LF, Marinho ACO, Gonçalves RT, Ferreira FB. Pseudoaneurisma pós-transplante renal - relato de caso. Rev Col Bras Cir. 2006; 33: 413-15.
9. Vine HS, Sacks BA: 'Visualization of the distal arterial vessels in complete aortic occlusion. AJR Am J Roentgenol. 1980; 134: 847-8.

10. Krupski WC, Sumchai A, Effeney DJ, Ehrenfeld WK: The importance of abdominal wall collateral blood vessels. Planning incisions and obtaining arteriography. Arch Surg. 1984; 119: 854-7.

Correspondence address:

Dr. Vanessa Prado dos Santos Instituto de Humanidades Artes e Ciências Professor Milton Santos Federal University, Bahia Rua Barão de Jeremoabo (PAF IV), S/N Campus de Ondina, Salvador, BA, 40170-115, Brazil

Telephone: + 5571 3283-6799

E-mail: vansanbr@hotmail.com 


\section{EDITORIAL COMMENT}

This is a rare case of a vascular enteric fistula presenting with massive rectal hemorrhage. As one may be unfortunate to run into such a challenging case, some points ought to be highlighted:

If diagnosis is suspected, immediate surgical aggressive management is required and should not be postponed;

A vascular surgeon presence is greatly appreciated as bleeding control may be very difficult;
Attempt to reconstruct the vessel is not the first option and ligation of the vessel is to be considered even when dealing with a terminal vessel such as the external iliac artery.

Dr. Ricardo Miyaoka Discipline Urology, UNICAMP Campus Universitario Zeferino Vaz SN Cidade Universitaria Campinas, 13083-970, SP, Brazil Telephone: + $55193521-7000$ E-mail: rmiyaoka@uol.com.br 